\title{
PROFITABILITY OF TRADING IN THE DIRECTION OF ASSET PRICE JUMPS - ANALYSIS OF MULTIPLE ASSETS AND FREQUENCIES
}

\section{Milan Fičura*}

\begin{abstract}
Profitability of a trading system based on the momentum-like effects of asset price jumps was tested on four currency markets (EUR/USD, GBP/USD, USD/CHF and USD/JPY) and three futures markets (Light Crude Oil, E-Mini S\&P 500 and VIX), on 7 frequencies (1-minute to 1-day), over a period of more than 20 years. The proposed trading system entered long and short trades in the direction of asset price jumps and held the positions for a fixed horizon, optimized on the insample period. The system achieved statistically significant out-sample profits for the USD/CHF, EUR/USD and GBP/USD exchange rates, especially on the 15-minute, 30-minute and 1-hour frequencies, with expected returns of up to $20-30 \%$ p.a., including transaction costs. On the 1-day frequency, on the USD/JPY and on the three analysed futures markets, only insignificant profits or losses were achieved. On the 1-minute frequency, the system ended with a loss for all of the assets.
\end{abstract}

Keywords: asset price jumps, L-estimator, high-frequency trading, momentum trading JEL Classification: C14, C58, G11, G14, G17

\section{Introduction}

A common practice in financial econometrics is to view asset price time series as governed by two sources of variability: a continuous stochastic volatility, and a discontinuous jump component. Jumps, representing large discontinuous price changes, can significantly increase the tails of the short-horizon return distribution, playing an important role in tasks such as option pricing, value-at-risk estimation or quantitative trading.

Advances in Bayesian estimation methods and stochastic-volatility jump-diffusion models, as well as in high-frequency data analysis and the asymptotic theory of power variations, have spurred a renewed interest among researchers in the area of jump modelling. Numerous studies analysing the jump dynamics emerged in the recent years, identifying effects such as jump self-exciting and clustering effects (Fulop et al., 2014; Fičura and Witzany, 2016), contagion effects between the jumps in different markets (Ait-Sahalia et al., 2015; Fičura, 2015), as well as positive impact of jumps on the future asset price volatility (Corsi et al., 2010; Bandi and Reno, 2016).

* Milan Fičura, Faculty of Finance and Accounting, University of Economics, Prague, Czech Republic (milan.ficura@vse.cz).

The paper has been prepared with financial support under the grant "Advanced methods of financial asset returns and risks modelling" IGA VŠE F1/23/2015, and the Czech Science Foundation Grant P402/12/G097 "Dynamical Models in Economics". 
Less research has focused on the idea of whether jumps may also have an impact on the expected mean of the future asset price returns. Among the papers that have pursued this possibility is the study of Behfar (2016), who found evidence of long-memory behaviour of the S\&P 500 Index after the occurrence of jumps, and the study of Novotny et al. (2015), analysing the profitability of an after-jump trading system on the 5-minute frequency on foreign exchange markets, finding that for the Euro, Yen and Rand, the strategy achieves positive profits, even in the presence of the bid-ask spreads.

As the jumps often coincide with macroeconomic news announcements, it is possible to link the momentum-like after-jump behaviour to a gradual or delayed reaction of asset prices to unexpected macroeconomic news. The evidence on whether prices react to macroeconomic news efficiently or in a delayed fashion is rather mixed, with studies of Brazys and Martens (2014) and Brazys et al. (2015) indicating a delayed reaction for the bond markets, while the study of Andersen et al. (2007), performed on a wide variety of bond, stock and foreign exchange markets, found no evidence of a statistically significant reaction beyond the first 5 minutes after the news announcement.

As the possible existence of a predictable after-jump behaviour of asset returns violates the weak form of the efficient market hypothesis, while at the same time being empirically interesting to practitioners, we perform a detailed analysis of this phenomenon on multiple assets and frequencies, in order to either confirm or reject the existence of the described momentum-like effects and evaluate whether they could be utilized to achieve speculative profits.

In the analysis performed, the after-jump behaviour is studied on seven different markets (EUR/USD, GBP/USD, USD/CHF and USD/JPY exchange rates, and the S\&P500, VIX and Light Crude Oil futures) over a time period of up to two decades, depending on the data availability for the given asset. The jumps are identified with a non-parametric $L$-estimator proposed by Lee and Mykland (2008), which identifies jumps at the exact time at which they occur and is thus particularly well suited for the purposes of this study. The analysis is performed for each of the assets on seven different frequencies, ranging from 1-minute to 1-day, in order to evaluate whether the possible inefficiencies exist only on the highest frequencies, or whether they persist on the lower frequencies as well.

To link the theoretical results of the study with a possible practical application, an after-jump momentum trading strategy is proposed, whose profitability is then evaluated in a methodologically robust way that avoids the risks of forward-looking bias, selection bias and overfitting. The data sample is divided into an in-sample and an out-sample period, with the in-sample period used to optimize the parameters of the proposed trading algorithm, while the out-sample period is used to evaluate the performance of the optimized models, with regard to their profitability, risk and statistical significance, in the presence of transaction costs.

The rest of the paper is organized as follows. In the first section, we explain the notion of realized bipower variation, used for local volatility estimation, and the $L$-estimator, used for non-parametric asset price jump identification. In the second section, the dataset utilized is presented and statistics about the identified jumps are discussed. In the third section, the after-jump trading strategy is proposed and its results are evaluated. 
Finally, in the conclusion, the results of the study are summarized and conclusions about the model performance are made.

\section{Non-parametric Jump Identification}

As the jumps in financial time series are inherently unobservable (we do not know whether a given large price movement was caused by a jump or not), it is necessary to estimate them from the observed returns with statistical methods. One possible approach is to use Bayesian estimation methods together with stochastic-volatility jump-diffusion models to model the asset price evolution and estimate the jumps as latent state variables (Ficura and Witzany, 2016). Another approach is to use high-frequency financial data and estimate the jumps with non-parametric jump estimators, derived by the asymptotic theory of power variations (Barndorff-Nielsen and Shephard, 2004). The benefit of the non-parametric approach is that it is far less time-consuming than the computationally intensive Bayesian methods, while at the same time being model-free, which means that the jump estimates are theoretically valid for a wide variety of possible price processes and not only for a single specific process as is the case with the parametric approach.

A large number of non-parametric jump estimators has been proposed in the recent years. The most commonly used are the Z-estimator (Barndorff-Nielsen and Shephard, 2004), L-estimator (Lee and Mykland, 2008) and the JO-estimator (Jiang and Oomen, 2008). Simulation studies comparing the performance of different non-parametric jump estimators can be found in Hanousek et al. (2011) or Dumitru and Urga (2012).

In our study, the jumps are identified with the $L$-estimator proposed by Lee and Mykland (2008), which shows a relatively strong performance in simulation tests, while at the same time identifying jumps at the exact time at which they occur, instead of identifying just whether any jumps occurred during a specified time period (as the $Z$-estimator does). This makes the $L$-estimator particularly suitable for our study, as we need to identify the jumps immediately as they occur.

The $L$-estimator uses returns normalized by local volatility, estimated with the realized bipower variation, to identify whether the given return was caused by a jump or not. Specifically, the method compares the size of the locally normalized returns with a certain quantile of the expected maximum of these normalized returns, with the distribution of the maximum derived under the assumption of no jumps in the time series. If the normalized return is larger than a sufficiently high quantile of the maximum return in a time series of the given length, then the return is identified as a jump.

To explain the $L$-estimator in more detail, it is necessary to define a general stochasticvolatility jump-diffusion process governing the evolution of the logarithmic returns of the asset price:

$$
d p(t)=\mu(t) d t+\sigma(t) d W(t)+j(t) d q(t),
$$

where $p(t)$ is the logarithm of the asset price, $\mu(t)$ is the instantaneous drift rate, $\sigma(t)$ is the instantaneous volatility, $W(t)$ is a Wiener process, $j(t)$ is a process determining the jump sizes and $q(t)$ is a counting process determining the jump occurrences. 
It is worth noting that in line with the model-free nature of the approach employed, the process in Equation 1 represents just a general specification of the jump-diffusion process of the price evolution, with the sub-processes governing $\mu(t), \sigma(t), j(t)$ and $q(t)$ being free to attain a wide variety of different forms.

The total variability of the process governing the asset price evolution over the period between $t-k$ and $t$ can then be expressed with its quadratic variation as follows:

$$
Q V(t-k, t)=\int_{t-k}^{t} \sigma^{2}(s) d s+\sum_{t-k \leq s<t} j^{2}(s) I[q(s)=1]
$$

where $Q V(t-k, t)$ denotes the quadratic variation for the period between $t-k$ and $t, \sigma^{2}(s)$ is the squared instantaneous variance, $j(t)$ are the jump sizes, $q(s)$ are the jump occurrences and $I($.$) is an indicator function. The first term on the right-hand side of the equation, \int_{t-k}^{t} \sigma^{2}(s) d s$, represents the continuous component of the asset price variability, called integrated variance, while the second term, $\sum_{t-k \leq s<t} j^{2}(s) I[q(s)=1]$, represents the aggregated impact of jumps (i.e., the discontinuous component of price variability), called jump variance. It is thus possible to rewrite the equation as follows:

$$
Q V(t-k, t)=I V(t-k, t)+J V(t-k, t),
$$

where $I V(t-k, t)$ is the integrated variance and $J V(t-k, t)$ is the jump variance.

The $L$-estimator (Lee and Mykland, 2008) proceeds to define local volatility as an approximate measure of the continuous price variability at a given time point of the time series. For this purpose, it uses the realized bipower variation $(B V)$, which represents an unbiased and asymptotically consistent estimator of the underlying integrated variance (IV) (Barndorff-Nielsen and Shephard, 2004). The local variance for time $i$ is then defined as the realized bipower variation of the price process over the last $k$ periods, with $k$ being a meta-parameter of the model:

$$
\sigma_{B V}^{2}(i)=B V(i-k, i-1)=\frac{1}{k-2} \sum_{j=i-k+2}^{i-1}|r(j)||r(j-1)|,
$$

where $\sigma_{B V}^{2}(i)$ is the local variance estimate for period $i, k$ is the number of periods used for local volatility estimation, and $r(j)$ is a logarithmic return at period $j$, defined as $r(j)=p(j)-p(j-1)$, where $p(j)$ is the logarithm of the price at end of period $j$. $B V(i-k, i-1)$ is the realized bipower variation over the period between $i-k$ and $i-1$, which converges, with increasing frequency of the returns used for the computation, to the integrated variance $I V(i-k, i-1)$.

The $L$-estimator can then be defined as follows:

$$
L(i)=\frac{r(i)}{\sigma_{B V}(i)},
$$

where $L(i)$ is the $L$-statistics, $r(i)$ is the return in time period $i$ and $\sigma_{B V}(i)$ is the local volatility, computed as a square root of the local variance $\sigma_{B V}^{2}(i)$. 
The method of jump identification based on the $L$-estimator uses the known distribution of the appropriately normalized maximum of the $L$-estimator in a time series $A_{n}$ of a length $n$, with the distribution derived under the assumption of no jumps in the time series. The normalized maximum can be expressed as:

$$
\xi=\frac{\max _{i \in A_{n}}|L(i)|-C_{n}}{S_{n}},
$$

where $A_{n}$ is the set of all periods in the analysed time series $i \in\{1,2, \ldots, n\}$, and $C_{n}$ and $S_{n}$ are constants defined as:

$$
\begin{gathered}
C_{n}=\frac{[2 \log (n)]^{1 / 2}}{c}-\frac{\log (\pi)+\log [\log (n)]}{2 c[2 \log (n)]^{1 / 2}} \\
S_{n}=\frac{1}{c[2 \log (n)]^{1 / 2}},
\end{gathered}
$$

with $c$ being a constant $c=\sqrt{2} / \sqrt{\pi} \approx 0.7979$.

To identify jumps in the time series, it is necessary to utilize the known distribution of the maximum $\xi$ in a time series of a length $n$, derived under the assumption of no jumps. The distribution of the maximum $\xi$ is:

$$
P(\xi \leq x)=\exp \left(-e^{-x}\right)
$$

Jumps can then be identified as the normalized values of $L(i)$, with the normalization performed based on Equation 6, that are larger than a given sufficiently high quantile $\alpha$ of the maximum $\xi$. The quantile $\alpha$ is commonly being chosen somewhere in the range between $\alpha=0.900$ and $\alpha=0.999$.

The jump estimation approach using the $L$-statistics has two meta-parameters: the period for local volatility calculation $k$, and the quantile for jump identification $\alpha$. Lee and Mykland (2008) derive optimality bounds for the selection of $k$ as $k_{\text {min }}=\sqrt{252 * \text { nobs }}$ and $k_{\max }=252 *$ nobs, with nobs being the number of observations during a trading day. Based on a simulation study, they find the optimal values of $k$ to be equal to the lower bound $k_{\min }$ and propose to use the values of $k$ equal to $7,16,78,110,156$ and 270 , for the frequencies of 1-week, 1-day, 1-hour, 30-minute, 15-minute and 5-minute respectively.

In our study, we use the values of $k$ proposed by Lee and Mykland (2008) for the mentioned frequencies, with the values for the 1-minute and 4-hour frequency (that are not mentioned in the original study), computed using the equation for $k_{\min }$.

\section{Dataset for the Jump Analysis}

The dataset used in the analysis contains time series with seven different frequencies (1-day, 4-hour, 1-hour, 30-minute, 15-minute, 5-minute and 1-minute), for four foreign exchange 
rates (EUR/USD, GBP/USD, USD/JPY and USD/CHF) and three futures contracts (E-Mini S\&P 500 Futures, VIX Futures and Light Crude Oil Futures). The futures time series are constructed as a continuous c1 contract, so that they represent the evolution of the futures prices always for the contract that is nearest to maturity. The currency exchange rate dataset ranges from 1 Nov 1999 to 12 Jun 2015, while the futures dataset range differs for each of the underlying assets. For E-Mini 500 futures it ranges from 11 Sep 1997 to 4 Dec 2015, for VIX futures from 26 Mar 2004 to 2 Dec 2015 and for Light Crude Oil futures from 2 Jan 1987 to 4 Dec 2015 . The foreign exchange rate dataset is provided by forexhistorydatabase. com and the futures dataset is provided by tickmarketdata.com.

Before implementing the analysis, the data were cleaned of the weekend gaps and the roll-over returns (for the futures time series), as these may manifest as illusory jumps that do not correspond to the swift discontinuous price changes which the study wants to examine.

The jumps in the time series are identified with the $L$-estimator of Lee and Mykland (2008). The parameter $k$ used for local volatility estimation is set to $k=\sqrt{252 * n o b s}$, with nobs representing the number of observations on the given frequency during a day. After rounding off the results to the nearest larger integer, the periods are given as $k=\{16,39,78$, $110,156,270,603\}$ for the frequencies $f=\{1 \mathrm{D}, 4 \mathrm{H}, 1 \mathrm{H}, 30 \mathrm{M}, 15 \mathrm{M}, 5 \mathrm{M}, 1 \mathrm{M}\}$.

Multiple confidence levels for jump identification (parameter $\alpha$, which will further be denoted as $p$ ) were tested and this parameter is optimized in the trading system that will be proposed. The confidence levels that were tested are $p=\{0.900,0.950,0.990,0.999\}$.

Table 1 and Table 2 show the identified numbers of jumps for all the analysed assets, frequencies and possible confidence levels.

We can see from Table 1 and Table 2 that the higher the return sampling frequency, the larger the identified number of jumps. This is to be expected as the discontinuous price changes become more visible at the higher frequencies than they are at the lower frequencies, at which they may often be indistinguishable from the continuous price variability.

Nevertheless, as argued in Ficura (2015), small high-frequency jumps are often caused by market microstructure noise effects, large market orders and insufficient liquidity in the order book, rather than unexpected macroeconomic news arrivals. The small intraday jumps do therefore have a far smaller effect on future price behaviour, especially regarding future volatility and jump intensity, than the large jumps, visible on the lower frequencies. Subsequently, we expect to see the largest trading profits of the proposed after-jump trading system rather at the medium frequencies, as the lowest ones contain too few jumps, while the highest ones contain far too many small uninformative jumps. 
Table 1 | Numbers of jumps identified by the L-estimator on different frequencies for the currency time series

\begin{tabular}{|c|c|c|c|c|c|c|}
\hline \multicolumn{2}{|c|}{ Analysed time series } & \multirow{2}{*}{ Observations } & \multicolumn{4}{|c|}{ Identified jumps for different confidence levels } \\
\hline Currency & Frequency & & $90 \%$ & $95 \%$ & $99 \%$ & $99.9 \%$ \\
\hline \multirow{7}{*}{ EUR/USD } & 1-day & 4,071 & 16 & 13 & 8 & 6 \\
\hline & 4-hour & 24,380 & 142 & 124 & 91 & 54 \\
\hline & 1-hour & 96,540 & 527 & 476 & 390 & 274 \\
\hline & 30-minute & 193,066 & 860 & 792 & 627 & 485 \\
\hline & 15-minute & 386,116 & 1,447 & 1,330 & 1,088 & 839 \\
\hline & 5-minute & $1,157,591$ & 3,337 & 3,083 & 2,595 & 2,049 \\
\hline & 1-minute & $5,770,678$ & 10,714 & 9,893 & 8,442 & 6,825 \\
\hline \multirow{7}{*}{ GBP/USD } & 1-day & 4,071 & 9 & 6 & 5 & 4 \\
\hline & 4-hour & 24,380 & 113 & 101 & 72 & 42 \\
\hline & 1-hour & 96,538 & 397 & 357 & 263 & 174 \\
\hline & 30-minute & 193,064 & 639 & 570 & 452 & 331 \\
\hline & 15-minute & 386,105 & 1,092 & 989 & 802 & 613 \\
\hline & 5-minute & $1,157,556$ & 2,624 & 2,391 & 1,951 & 1,537 \\
\hline & 1-minute & $5,770,406$ & 10,678 & 9,846 & 8,233 & 6,586 \\
\hline \multirow{7}{*}{ USD/CHF } & 1-day & 4,070 & 13 & 11 & 10 & 7 \\
\hline & 4-hour & 24,377 & 151 & 138 & 105 & 73 \\
\hline & 1-hour & 96,520 & 528 & 469 & 386 & 286 \\
\hline & 30-minute & 193,027 & 872 & 792 & 651 & 506 \\
\hline & 15-minute & 386,015 & 1,443 & 1,304 & 1,102 & 846 \\
\hline & 5-minute & $1,157,387$ & 3,405 & 3,122 & 2,602 & 2,041 \\
\hline & 1-minute & $5,779,133$ & 11,346 & 10,493 & 8,913 & 7,176 \\
\hline \multirow{7}{*}{ USD/JPY } & 1-day & 4,071 & 28 & 23 & 20 & 12 \\
\hline & 4-hour & 24,380 & 149 & 131 & 94 & 69 \\
\hline & 1-hour & 96,540 & 463 & 409 & 337 & 251 \\
\hline & 30-minute & 193,067 & 823 & 748 & 594 & 441 \\
\hline & 15-minute & 386,112 & 1,299 & 1,171 & 974 & 755 \\
\hline & 5-minute & $1,157,560$ & 2,897 & 2,676 & 2,258 & 1,798 \\
\hline & 1-minute & $5,769,309$ & 8,608 & 8,055 & 6,876 & 5,646 \\
\hline
\end{tabular}

Source: Authorial computation using data from forexhistorydatabase.com 
Table 2 | Numbers of jumps identified by the $L$-estimator on different frequencies for the futures time series

\begin{tabular}{|c|c|c|c|c|c|c|}
\hline \multicolumn{2}{|c|}{ Analysed time series } & \multirow{2}{*}{ Observations } & \multicolumn{4}{|c|}{ Identified jumps for different confidence levels } \\
\hline Currency & Frequency & & $90 \%$ & $95 \%$ & $99 \%$ & $99.9 \%$ \\
\hline \multirow{7}{*}{ Crude Oil } & 1-day & 2,547 & 14 & 14 & 12 & 9 \\
\hline & 4-hour & 17,729 & 110 & 92 & 63 & 41 \\
\hline & 1-hour & 74,018 & 466 & 407 & 315 & 230 \\
\hline & 30-minute & 146,337 & 981 & 882 & 733 & 582 \\
\hline & 15-minute & 288,232 & 1,865 & 1,738 & 1,444 & 1,147 \\
\hline & 5-minute & 867,718 & 3,410 & 3,193 & 2,773 & 2,309 \\
\hline & 1-minute & $4,343,829$ & 12,808 & 11,957 & 10,325 & 8,482 \\
\hline \multirow{7}{*}{ S\&P 500} & 1-day & 4,521 & 24 & 22 & 9 & 4 \\
\hline & 4-hour & 18,876 & 84 & 76 & 53 & 32 \\
\hline & 1-hour & 84,849 & 344 & 302 & 235 & 155 \\
\hline & 30-minute & 166,977 & 630 & 561 & 425 & 304 \\
\hline & 15-minute & 336,718 & 1,132 & 1,020 & 794 & 572 \\
\hline & 5-minute & $1,008,570$ & 2,405 & 2,193 & 1,743 & 1,350 \\
\hline & 1-minute & $5,045,077$ & 11,508 & 10,472 & 8,544 & 6,535 \\
\hline \multirow{7}{*}{ VIX } & 1-day & 1,582 & 25 & 23 & 16 & 13 \\
\hline & 4-hour & 2,705 & 24 & 23 & 19 & 13 \\
\hline & 1-hour & 10,946 & 91 & 83 & 64 & 46 \\
\hline & 30-minute & 21,550 & 140 & 127 & 110 & 81 \\
\hline & 15-minute & 44,342 & 234 & 209 & 177 & 136 \\
\hline & 5-minute & 131,426 & 478 & 448 & 380 & 313 \\
\hline & 1-minute & 658,299 & 1,160 & 1,083 & 940 & 803 \\
\hline
\end{tabular}

Source: Authorial computation using data from tickmarketdata.com

\section{After-jump Trading Strategy Analysis}

To evaluate whether jumps do have a momentum-like impact on subsequent returns, an after-jump momentum trading system is proposed, and its profitability is analysed.

The proposed algorithm enters a trade in the direction of the asset price jump, immediately after the jump is identified with the $L$-estimator at a confidence level $p$. The entry is assumed to be realized at the closing price of the period and the position is 
held for $h$ periods into the future, when it is closed. The holding period $h$, together with the confidence level for jump identification $p$, are meta-parameters of the model that are going to be optimized on the in-sample period to maximize the model performance. The period for local volatility estimation $k$, which is a third parameter of the model, will not be optimized but will instead be set to the recommended values according to the formula from Lee and Mykland (2008) mentioned in the previous section.

The meta-parameters will be optimized on the in-sample period with a simple grid search, with the tested values being $p=\{0.900,0.950,0.990,0.999\}$ and $h=\{1,2,3, \ldots, 24\}$. The in-sample period will, for each of the time series, correspond to the first $50 \%$ of the available observations, while the second half will be used as the out-sample period to evaluate the model performance and statistical significance.

The statistical significance of the profits is assessed using the $t$-test, testing whether the mean return of the strategy, during the periods in which a position was held, is significantly larger than zero. The formula of the $t$-test is given by $t=\frac{\bar{r}}{\left(\sigma_{r} / \sqrt{n}\right)}$, with $\bar{r}$ denoting the average return of the strategy, $\sigma_{r}$ the standard deviation of the returns and $n$ the number of periods during which a position was held. Under the null hypothesis of zero mean return, the test statistics follow Student's $t$-distribution with $n-1$ degrees of freedom, which enables us to evaluate the statistical significance of the attained values. The $p$-value of the $t$-test will be used for the statistical validation of the out-sample results, as well as for the in-sample optimization of the model parameters $p$ and $h$.

The profits of the system are calculated under the assumption of a fixed position size of 1 lot for the currencies or 1 contract for the futures. Fixed transaction costs (spreads and commissions) are assumed in both cases, equal to 1 pip for the currency time series (equal to 10 USD for the EUR/USD) and 1 tick + broker commission for the futures time series. The broker commission for futures is set to 2.2 USD per contract and the spreads are assumed to be one tick wide, corresponding to 10 USD for Light Crude Oil, 12.5 USD for the E-Mini S\&P 500 and 50 USD for the VIX Futures. These transaction costs are included in all the calculations, including the calculations of the final profits, as well as the statistical tests.

In addition to the total profit of the system, the maximum historical drawdown (max DD) will be calculated as well, defined as the maximum decrease in equity over the whole trading period (in-sample or out-sample). From the total profit and the maximum drawdown, the drawdown ratio (DD ratio) can be calculated, as the ratio between the average profit p.a. and the maximum historical drawdown. The drawdown ratio represents a widely used measure of risk-adjusted performance, which can also be used to estimate the expected annual return of the system in the case of leverage utilization. As the foreign exchange and futures markets enable the speculators to use relatively high levels of leverage, the capital required to trade a given strategy is often calculated based on its historical drawdowns. A commonly used rule is to estimate the capital required for the strategy as the sum of the margin requirement set by the broker and the maximum historical drawdown of the strategy, which assures that the investor will be able to continue trading even if the maximum historical drawdown occurs immediately at the beginning of the trading period. 
Table 3 | Profitability of the after-jump momentum trading system applied to the currency exchange rates (in-sample)

\begin{tabular}{|c|c|c|c|c|c|c|c|c|c|c|c|}
\hline \multicolumn{2}{|c|}{ Analysed series } & \multicolumn{3}{|c|}{ Model parameters } & \multicolumn{7}{|c|}{ In-sample results } \\
\hline Currency & Frequency & $\begin{array}{l}k \\
\text { fix }\end{array}$ & p_opt & $\begin{array}{l}h_{-} \\
\text {opt }\end{array}$ & periods & trades & profit & $\max D D$ & $\begin{array}{c}\text { DD } \\
\text { ratio }\end{array}$ & t-stat & $p$-value \\
\hline \multirow{7}{*}{ EUR/USD } & 1-day & 16 & 0.999 & 8 & 2,035 & 2 & 2,130 & -780 & 0.338 & 0.991 & 0.168 \\
\hline & 4-hour & 39 & 0.950 & 11 & 12,190 & 69 & 11,830 & $-4,630$ & 0.316 & 1.780 & 0.038 \\
\hline & 1-hour & 78 & 0.900 & 2 & 48,270 & 253 & 13,650 & $-2,750$ & 0.615 & 2.883 & 0.002 \\
\hline & 30-minute & 110 & 0.900 & 4 & 96,533 & 408 & 12,820 & $-10,185$ & 0.156 & 2.244 & 0.012 \\
\hline & 15-minute & 156 & 0.900 & 9 & 193,058 & 650 & 11,400 & $-5,360$ & 0.263 & 1.554 & 0.060 \\
\hline & 5-minute & 270 & 0.999 & 11 & 578,795 & 862 & $-7,660$ & $-13,830$ & -0.069 & -0.392 & 0.653 \\
\hline & 1-minute & 603 & 0.999 & 1 & $2,885,339$ & 2,404 & $-47,210$ & $-47,210$ & -0.124 & -6.952 & 1.000 \\
\hline \multirow{7}{*}{ GBP/USD } & 1-day & 16 & 0.999 & 19 & 2,035 & 2 & 12,310 & $-1,220$ & 1.249 & 2.402 & 0.011 \\
\hline & 4-hour & 39 & 0.900 & 10 & 12,190 & 51 & 16,330 & $-4,950$ & 0.409 & 2.121 & 0.017 \\
\hline & 1-hour & 78 & 0.999 & 21 & 48,269 & 78 & 24,490 & $-3,550$ & 0.854 & 3.302 & 0.000 \\
\hline & 30-minute & 110 & 0.990 & 3 & 96,532 & 201 & 11,730 & $-2,930$ & 0.496 & 2.500 & 0.006 \\
\hline & 15-minute & 156 & 0.990 & 12 & 193,052 & 347 & 15,340 & $-11,320$ & 0.168 & 1.852 & 0.032 \\
\hline & 5-minute & 270 & 0.999 & 24 & 578,778 & 612 & 1,070 & $-17,560$ & 0.008 & 0.315 & 0.377 \\
\hline & 1-minute & 603 & 0.999 & 1 & $2,885,203$ & 2,203 & $-64,840$ & $-64,840$ & -0.124 & -9.820 & 1.000 \\
\hline \multirow{7}{*}{ USD/CHF } & 1-day & 16 & 0.900 & 5 & 2,035 & 5 & 2,887 & -996 & 0.359 & 1.369 & 0.092 \\
\hline & 4-hour & 39 & 0.990 & 11 & 12,188 & 53 & 8,995 & $-1,394$ & 0.799 & 2.238 & 0.013 \\
\hline & 1-hour & 78 & 0.950 & 2 & 48,260 & 221 & 8,652 & $-2,498$ & 0.429 & 2.819 & 0.003 \\
\hline & 30-minute & 110 & 0.950 & 2 & 96,513 & 372 & 9,011 & $-2,443$ & 0.457 & 3.100 & 0.001 \\
\hline & 15-minute & 156 & 0.900 & 6 & 193,007 & 653 & 7,007 & $-3,418$ & 0.254 & 1.731 & 0.042 \\
\hline & 5-minute & 270 & 0.999 & 23 & 578,693 & 810 & $-2,761$ & $-12,956$ & -0.026 & 0.114 & 0.455 \\
\hline & 1-minute & 603 & 0.999 & 1 & $2,889,566$ & 2,651 & $-48,458$ & $-48,458$ & -0.124 & -12.476 & 1.000 \\
\hline \multirow{7}{*}{ USD/JPY } & 1-day & 16 & 0.900 & 14 & 2,035 & 14 & 5,871 & $-8,417$ & 0.086 & 0.826 & 0.205 \\
\hline & 4-hour & 39 & 0.900 & 23 & 12,190 & 60 & 3,458 & $-6,732$ & 0.064 & 0.433 & 0.332 \\
\hline & 1-hour & 78 & 0.990 & 2 & 48,270 & 148 & 2,091 & $-2,140$ & 0.121 & 0.857 & 0.196 \\
\hline & 30-minute & 110 & 0.999 & 6 & 96,533 & 185 & 7,924 & $-3,117$ & 0.315 & 2.070 & 0.019 \\
\hline & 15-minute & 156 & 0.900 & 9 & 193,056 & 543 & 7,897 & $-4,699$ & 0.208 & 1.549 & 0.061 \\
\hline & 5-minute & 270 & 0.999 & 9 & 578,780 & 719 & $-22,260$ & $-27,387$ & -0.101 & -2.938 & 0.998 \\
\hline & 1-minute & 603 & 0.999 & 1 & $2,884,654$ & 2,290 & $-43,017$ & $-43,021$ & -0.124 & -9.178 & 1.000 \\
\hline
\end{tabular}

Source: Authorial computation using data from forexhistorydatabase.com 
As the margin requirements on foreign exchange markets vary for different brokers (ranging from 1:50 up to 1:500), we use the simplified rule for required capital estimation and set the capital requirement to double the maximum historical drawdown. The expected annual return of the system would then be approximately equal to one half of the drawdown ratio.

Table 3 and Table 4 show the results of the trading system for the currency markets in the in-sample and the out-sample periods. The colours in the tables range from green to red, with green representing the relatively best values and red the worst ones.

The last 5 columns of the tables show the results of the model, namely the total profit in USD (profit), maximum historical drawdown (max DD), the drawdown ratio (DD ratio), the test value ( $t$-stat) of the $t$-test and the $p$-value of the t-test, testing whether the mean return is significantly larger than zero.

As can be seen from the last column of Table 3 and Table 4, the $p$-values of the $t$-test show high levels of statistical significance $(<1 \%)$ for some of the achieved profits, in the insample as well as the out-sample period. While the in-sample results are arguably biased due to the optimization performed, the out-sample results are unbiased, thus representing a strong evidence for the existence of momentum-like after-jump effects in some of the time series analysed.

The highest statistical significance and highest out-sample profits were achieved on the EUR/USD and USD/CHF exchange rates at the medium frequencies, followed by the GBP/USD, whose profits have a lower statistical significance, while the USD/JPY achieved mostly insignificant profits or losses.

The time frequencies with most statistically significant profits in the out-sample period are the medium ones, ranging between 5-minute and 4-hour, with the best results achieved on the 15-minute, 30-minute and 1-hour frequency.

The 1-day frequency contains too few jumps to make any strong conclusions, but the profits are generally low and insignificant, while two of the currencies achieved a loss. This indicates that the markets may already be efficient at this frequency.

The 1-minute frequency achieved significant losses for all of the currencies and it seems that it might even be profitable to enter into reverse, mean-reverting positions, instead of the momentum ones. The profitability of this approach would, however, need to be verified in a separate study, which would take into account the potentially higher transaction costs from trading on the 1-minute frequency than what could be expected from the lower-frequency trading.

A possible explanation as to why the 1-minute frequency behaves differently from all the others could be that the jumps on this frequency tend to be caused by microstructure noise effects, such as temporary liquidity insufficiencies, rather than arrivals of unexpected news. This may lead to an after-jump behaviour opposite to what we observe on the other frequencies. 
Table 4 | Profitability of the after-jump momentum trading system applied to the currency exchange rates (out-sample)

\begin{tabular}{|c|c|c|c|c|c|c|c|c|c|c|c|}
\hline \multicolumn{2}{|c|}{ Analysed series } & \multicolumn{3}{|c|}{$\begin{array}{c}\text { Model } \\
\text { parameters }\end{array}$} & \multicolumn{7}{|c|}{ Out-sample results } \\
\hline Currency & Frequency & $\begin{array}{l}k \\
\text { fix }\end{array}$ & $\begin{array}{l}p_{-} \\
\text {opt }\end{array}$ & $\begin{array}{l}\text { h_ } \\
\text { opt }\end{array}$ & periods & trades & profit & $\begin{array}{c}\max \\
\mathrm{DD}\end{array}$ & $\begin{array}{c}\text { DD } \\
\text { ratio }\end{array}$ & t-stat & $p$-value \\
\hline \multirow{7}{*}{ EUR/USD } & 1-day & 16 & 0.999 & 8 & 2,036 & 3 & 1,779 & -994 & 0.222 & 0.737 & 0.234 \\
\hline & 4-hour & 39 & 0.950 & 11 & 12,190 & 52 & 12,824 & $-3,869$ & 0.410 & 1.918 & 0.028 \\
\hline & 1-hour & 78 & 0.900 & 2 & 48,270 & 260 & 10,460 & $-2,650$ & 0.489 & 1.997 & 0.023 \\
\hline & 30-minute & 110 & 0.900 & 4 & 96,533 & 414 & 17,010 & $-4,790$ & 0.440 & 2.590 & 0.005 \\
\hline & 15-minute & 156 & 0.900 & 9 & 193,058 & 674 & 25,304 & $-6,283$ & 0.498 & 2.828 & 0.002 \\
\hline & 5-minute & 270 & 0.999 & 11 & 578,796 & 987 & 15,509 & $-6,339$ & 0.303 & 2.116 & 0.017 \\
\hline & 1-minute & 603 & 0.999 & 1 & $2,885,339$ & 4,157 & $-61,497$ & $-62,162$ & -0.122 & -7.244 & 1.000 \\
\hline \multirow{7}{*}{ GBP/USD } & 1-day & 16 & 0.999 & 19 & 2,036 & 1 & 1,306 & $-3,450$ & 0.047 & 0.360 & 0.362 \\
\hline & 4-hour & 39 & 0.900 & 10 & 12,190 & 59 & 10,053 & $-9,290$ & 0.134 & 0.981 & 0.164 \\
\hline & 1-hour & 78 & 0.999 & 21 & 48,269 & 92 & 17,610 & $-5,080$ & 0.429 & 1.826 & 0.034 \\
\hline & 30-minute & 110 & 0.990 & 3 & 96,532 & 237 & 7,517 & $-4,989$ & 0.186 & 1.470 & 0.071 \\
\hline & 15-minute & 156 & 0.990 & 12 & 193,053 & 414 & 15,542 & $-6,975$ & 0.276 & 1.811 & 0.035 \\
\hline & 5-minute & 270 & 0.999 & 24 & 578,778 & 780 & 10,359 & $-12,495$ & 0.103 & 1.115 & 0.133 \\
\hline & 1-minute & 603 & 0.999 & 1 & $2,885,203$ & 4,146 & $-76,235$ & $-76,320$ & -0.124 & -8.977 & 1.000 \\
\hline \multirow{7}{*}{ USD/CHF } & 1-day & 16 & 0.900 & 5 & 2,035 & 7 & $-18,392$ & $-21,002$ & -0.108 & -1.963 & 0.971 \\
\hline & 4-hour & 39 & 0.990 & 11 & 12,189 & 49 & 19,430 & $-6,788$ & 0.354 & 1.105 & 0.135 \\
\hline & 1-hour & 78 & 0.950 & 2 & 48,260 & 228 & 19,537 & $-6,317$ & 0.383 & 1.545 & 0.061 \\
\hline & 30-minute & 110 & 0.950 & 2 & 96,514 & 380 & 22,193 & $-2,373$ & 1.158 & 1.823 & 0.034 \\
\hline & 15-minute & 156 & 0.900 & 6 & 193,008 & 669 & 34,998 & $-4,193$ & 1.034 & 2.209 & 0.014 \\
\hline & 5-minute & 270 & 0.999 & 23 & 578,694 & 962 & 21,113 & $-10,689$ & 0.245 & 1.410 & 0.079 \\
\hline & 1-minute & 603 & 0.999 & 1 & $2,889,567$ & 4,264 & $-54,139$ & $-54,416$ & -0.123 & -5.904 & 1.000 \\
\hline \multirow{7}{*}{ USD/JPY } & 1-day & 16 & 0.900 & 14 & 2,036 & 13 & $-11,207$ & $-26,055$ & -0.053 & -1.258 & 0.895 \\
\hline & 4-hour & 39 & 0.900 & 23 & 12,190 & 79 & $-9,870$ & $-26,314$ & -0.046 & -0.615 & 0.731 \\
\hline & 1-hour & 78 & 0.990 & 2 & 48,270 & 178 & 1,230 & $-6,874$ & 0.022 & 0.331 & 0.370 \\
\hline & 30-minute & 110 & 0.999 & 6 & 96,534 & 231 & 5,221 & $-9,465$ & 0.068 & 0.838 & 0.201 \\
\hline & 15-minute & 156 & 0.900 & 9 & 193,056 & 636 & 7,095 & $-10,337$ & 0.085 & 0.920 & 0.179 \\
\hline & 5-minute & 270 & 0.999 & 9 & 578,780 & 923 & $-17,673$ & $-19,755$ & -0.111 & -1.367 & 0.914 \\
\hline & 1-minute & 603 & 0.999 & 1 & $2,884,655$ & 3,111 & $-52,164$ & $-52,699$ & -0.123 & -5.670 & 1.000 \\
\hline
\end{tabular}

Source: Authorial computation using data from forexhistorydatabase.com 
As the proposed system clearly achieved statistically significant profits on some of the frequencies, the next step is to evaluate what potential returns this may offer to investors. For this we can use the leverage rule, demanding that the trading capital covers at least twice the maximum drawdown, which would cause the expected return to be equal to approximately one half of the drawdown ratio. The potential out-sample returns for the medium frequencies (15-minute, 30-minute and 1-hour) on EUR/USD would thus be around 20-25\% p.a., for the GBP/USD around 10-20\% p.a., for the USD/CHF up to 50\% p.a., and for the USD/JPY around 2-4\% p.a.

Table 5 | Profitability of the after-jump momentum trading system applied to the futures markets (in-sample)

\begin{tabular}{|c|c|c|c|c|c|c|c|c|c|c|c|}
\hline \multicolumn{2}{|c|}{ Analysed series } & \multicolumn{3}{|c|}{ Model parameters } & \multicolumn{7}{|c|}{ In-sample results } \\
\hline 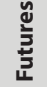 & Frequency & k_fix & p_opt & h_opt & periods & trades & profit & $\max D D$ & $\begin{array}{l}\text { DD } \\
\text { ratio }\end{array}$ & t-stat & $p$-value \\
\hline \multirow{7}{*}{ 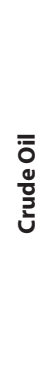 } & 1-day & 16 & 0.990 & 6 & 1,273 & 8 & 53,005 & $-9,342$ & 1.123 & 2.074 & 0.022 \\
\hline & 4-hour & 39 & 0.990 & 8 & 8,864 & 20 & 1,712 & $-10,094$ & 0.034 & 0.204 & 0.419 \\
\hline & 1-hour & 78 & 0.900 & 5 & 37,009 & 274 & $-17,776$ & $-24,060$ & -0.146 & -1.032 & 0.849 \\
\hline & 30-minute & 110 & 0.950 & 1 & 73,168 & 585 & $-12,674$ & $-19,260$ & -0.130 & -1.040 & 0.851 \\
\hline & 15-minute & 156 & 0.990 & 24 & 144,116 & 884 & $-16,000$ & $-45,128$ & -0.070 & -0.396 & 0.654 \\
\hline & 5-minute & 270 & 0.999 & 13 & 433,859 & 1,587 & $-30,733$ & $-35,734$ & -0.170 & -1.184 & 0.882 \\
\hline & 1-minute & 603 & 0.999 & 23 & $2,171,914$ & 4,139 & $-77,482$ & $-80,984$ & -0.189 & -2.196 & 0.986 \\
\hline \multirow{7}{*}{ 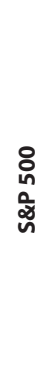 } & 1-day & 16 & 0.999 & 17 & 2,260 & 2 & -146 & $-5,700$ & -0.003 & -0.026 & 0.510 \\
\hline & 4-hour & 39 & 0.999 & 2 & 9,438 & 19 & 929 & $-1,448$ & 0.072 & 0.440 & 0.331 \\
\hline & 1-hour & 78 & 0.990 & 17 & 42,424 & 105 & 6,251 & $-9,341$ & 0.075 & 0.951 & 0.171 \\
\hline & 30-minute & 110 & 0.990 & 4 & 83,488 & 201 & 3,591 & $-4,912$ & 0.082 & 0.854 & 0.197 \\
\hline & 15-minute & 156 & 0.990 & 4 & 168,359 & 316 & 6,972 & $-5,662$ & 0.137 & 1.483 & 0.069 \\
\hline & 5-minute & 270 & 0.999 & 6 & 504,285 & 593 & $-4,972$ & $-7,618$ & -0.073 & -0.029 & 0.511 \\
\hline & 1-minute & 603 & 0.999 & 19 & $2,522,538$ & 2,903 & $-58,486$ & $-60,955$ & -0.107 & -3.737 & 1.000 \\
\hline \multirow{7}{*}{$\stackrel{x}{\bar{y}}$} & 1-day & 16 & 0.999 & 4 & 791 & 6 & 10,594 & $-1,354$ & 2.492 & 1.802 & 0.042 \\
\hline & 4-hour & 39 & 0.900 & 4 & 1,352 & 12 & -403 & $-8,256$ & -0.016 & -0.010 & 0.504 \\
\hline & 1-hour & 78 & 0.950 & 14 & 5,473 & 53 & $-27,093$ & $-33,508$ & -0.258 & -2.084 & 0.981 \\
\hline & 30-minute & 110 & 0.900 & 1 & 10,775 & 93 & $-4,869$ & $-5,474$ & -0.283 & -0.746 & 0.771 \\
\hline & 15-minute & 156 & 0.999 & 13 & 22,171 & 88 & 5,383 & $-12,704$ & 0.135 & 0.452 & 0.326 \\
\hline & 5-minute & 270 & 0.999 & 8 & 65,713 & 232 & 6,609 & $-6,996$ & 0.301 & 1.246 & 0.107 \\
\hline & 1-minute & 603 & 0.999 & 20 & 329,149 & 633 & $-98,145$ & $-99,022$ & -0.316 & -4.629 & 1.000 \\
\hline
\end{tabular}

Source: Authorial computation using data from tickmarketdata.com 
It is worth noting that the USD/CHF out-sample returns were probably significantly influenced by the turmoil on the market after the introduction and subsequent end of the Swiss National Bank monetary interventions, which both happened during the outsample period. The expected future returns would therefore be most probably lower than what was observed in the past.

Table 6 | Profitability of the after-jump momentum trading system applied to the futures markets (out-sample)

\begin{tabular}{|c|c|c|c|c|c|c|c|c|c|c|c|}
\hline \multicolumn{2}{|c|}{ Analysed series } & \multicolumn{3}{|c|}{ Model parameters } & \multicolumn{7}{|c|}{ Out-sample results } \\
\hline 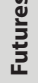 & Frequency & k_opt & p_opt & h_opt & periods & trades & profit & $\max D D$ & $\begin{array}{l}\text { D D } \\
\text { ratio }\end{array}$ & $t$-stat & $p$-value \\
\hline \multirow{7}{*}{ 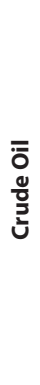 } & 1-day & 16 & 0.990 & 6 & 1,274 & 3 & 5,587 & $-6,080$ & 0.182 & 0.623 & 0.271 \\
\hline & 4-hour & 39 & 0.990 & 8 & 8,865 & 36 & 20,392 & $-6,896$ & 0.585 & 1.454 & 0.073 \\
\hline & 1-hour & 78 & 0.900 & 5 & 37,009 & 185 & $-13,354$ & $-23,646$ & -0.112 & -1.075 & 0.859 \\
\hline & 30-minute & 110 & 0.950 & 1 & 73,169 & 287 & 127 & $-5,034$ & 0.005 & 0.343 & 0.366 \\
\hline & 15-minute & 156 & 0.990 & 24 & 144,116 & 439 & $-37,192$ & $-50,596$ & -0.145 & -1.721 & 0.957 \\
\hline & 5-minute & 270 & 0.999 & 13 & 433,859 & 619 & -3734 & $-17,996$ & -0.041 & 0.036 & 0.485 \\
\hline & 1-minute & 603 & 0.999 & 23 & $2,171,915$ & 3305 & $-35,882$ & $-37,312$ & -0.190 & -0.693 & 0.756 \\
\hline \multirow{7}{*}{$\begin{array}{l}\text { 우 } \\
\text { ñ } \\
\text { ઝ } \\
\text { n }\end{array}$} & 1-day & 16 & 0.999 & 17 & 2,261 & 2 & $-7,259$ & $-8,425$ & -0.096 & -1.112 & 0.863 \\
\hline & 4-hour & 39 & 0.999 & 2 & 9,438 & 13 & 1,655 & -509 & 0.363 & 1.236 & 0.114 \\
\hline & 1-hour & 78 & 0.990 & 17 & 42,425 & 123 & $-10,016$ & $-10,703$ & -0.104 & -1.005 & 0.843 \\
\hline & 30-minute & 110 & 0.990 & 4 & 83,489 & 208 & -503 & $-6,186$ & -0.009 & 0.242 & 0.404 \\
\hline & 15-minute & 156 & 0.990 & 4 & 168,359 & 437 & $-16,735$ & $-18,056$ & -0.103 & -2.024 & 0.978 \\
\hline & 5-minute & 270 & 0.999 & 6 & 504,285 & 656 & $-6,649$ & $-10,370$ & -0.071 & -0.188 & 0.574 \\
\hline & 1-minute & 603 & 0.999 & 19 & $2,522,539$ & 2626 & $-45,617$ & $-47,550$ & -0.107 & -2.449 & 0.993 \\
\hline \multirow{7}{*}{$\stackrel{\times}{>}$} & 1-day & 16 & 0.999 & 4 & 791 & 7 & $-3,481$ & $-5,904$ & -0.188 & -0.684 & 0.750 \\
\hline & 4-hour & 39 & 0.900 & 4 & 1,353 & 12 & 1,737 & $-2,722$ & 0.203 & 0.367 & 0.358 \\
\hline & 1-hour & 78 & 0.950 & 14 & 5,473 & 29 & $-12,988$ & $-13,578$ & -0.305 & -1.868 & 0.969 \\
\hline & 30-minute & 110 & 0.900 & 1 & 10,775 & 46 & $-3,972$ & $-4,095$ & -0.309 & -1.623 & 0.944 \\
\hline & 15-minute & 156 & 0.999 & 13 & 22,171 & 45 & $-6,478$ & $-9,542$ & -0.216 & -1.389 & 0.917 \\
\hline & 5-minute & 270 & 0.999 & 8 & 65,713 & 66 & 1,550 & $-2,006$ & 0.246 & 0.853 & 0.197 \\
\hline & 1-minute & 603 & 0.999 & 20 & 329,150 & 140 & $-16,696$ & $-17,220$ & -0.309 & -3.567 & 1.000 \\
\hline
\end{tabular}

Source: Authorial computation using data from tickmarketdata.com 
Table 5 and Table 6 show the results for the futures contracts analysed.

It is apparent that unlike the currency markets, the model achieved only poor results on the futures markets, with relatively low statistical significance of the profits, even in the in-sample period. In the out-sample period, the system achieved mostly losses or insignificant profits, with none of the $p$-values falling below the $5 \%$ confidence level.

The best results were achieved on the 4-hour frequency, which turned out to be profitable for all of the futures contracts analysed. The profits on Light Crude Oil have the highest statistical significance ( $p$-value of $7.3 \%$ ), with expected profits of up to $25-30 \%$ p.a. (derived from the drawdown ratio).

Overall, the results for the futures markets do not support the hypothesis of a momentumlike price behaviour after jump occurrences. A reason for this may be that all of the analysed markets contain relatively high risk premiums (positive for S\&P 500, negative for VIX and time-varying for the Light Crude Oil). As the occurrence of a jump tends to increase the market risk premiums substantially, a mean-reverting behaviour can be expected after negative jumps in S\&P 500 or positive jumps in the VIX. This goes against our assumed momentum-like effects associated with gradual reaction of the markets to new unexpected information, and it may thus cause the after-jump behaviour of these assets to be more ambiguous than in the case of the currency markets.

\section{Conclusion}

Profitability of a trading system based on the momentum-like effects of price jumps was tested on the time series of seven different assets (EUR/USD, GBP/USD, USD/CHF and USD/JPY exchange rates and Light Crude Oil, E-Mini S\&P 500 and VIX futures), in each case for seven different frequencies (1-minute, 5-minute, 15-minute, 30-minute, 1-hour, 4-hour and 1-day), while taking into account the transaction costs (1 pip for the currencies and 1 tick spread + broker commission for the futures).

The trading system entered into long and short positions in the direction of price jumps, immediately after the jumps were identified with the non-parametric $L$-estimator. The position was then held for the following 1 to 24 periods.

Meta-parameters of the model (confidence level for jump identification and the holding period) were optimized on the in-sample period (first $50 \%$ of the data), while the performance of the optimized models was then evaluated on the out-sample period (remaining 50\% of the data). The optimization was performed with the goal of minimizing the $p$-value of the in-sample $t$-test, testing whether the mean value of model returns is significantly larger than zero.

The results of the study show that the after-jump momentum trading system achieves statistically significant out-sample profits on the EUR/USD, GBP/USD and USD/CHF currencies on most of the tested frequencies, with the exception of the 1-day and 1-minute ones. The most suitable frequencies for trading, with the most statistically significant outsample profits, are the 15-minute, 30-minute and 1-hour ones, with expected returns of 20$25 \%$ p.a. for EUR/USD, 10-20\% p.a. for GBP/USD, and up to 50\% p.a. for USD/CHF. 
The USD/JPY, on the other hand, achieved mostly insignificant profits or losses in the outsample period, with expected returns being at most $2-4 \%$ p.a.

The results for the 1-day frequency were not particularly good and even the profits achieved were not statistically significant, indicating that the markets may be efficient at this frequency. Similarly, the 1-minute frequency achieved a significant loss for all of the currencies, which can be attributed to different properties of the jumps on the ultrahigh frequencies, as they tend to be caused by market microstructure noise rather than unexpected news arrivals, which may result in a mean-reverting reaction instead of a momentum-like one.

The analysis performed on the futures markets led to dramatically different results as the system did not achieve statistically significant profits on any of the tested time series, and on most of them it ended up with a loss. This could possibly be attributed to the effects of the jumps on the risk premiums on these markets, which may lead rather to a meanreverting behaviour after the occurrence of jumps.

The conclusion is that while statistically significant momentum-like after-jump behaviour was confirmed on the 5-minute to 4-hour frequencies on the currency markets (EUR/USD, GBP/USD and USD/CHF), it does not seem to be present on the 1-minute and the 1-day frequencies, it is only weakly present for the USD/JPY exchange rate, and it does not seem to be present on any of the futures markets analysed (Light Crude Oil, S\&P 500 and VIX).

The reasons why certain markets behave differently than others, and whether it is because they are efficient or because of different kinds of inefficiencies (such as meanreversion) remains an open question which poses an interesting area for future research.

\section{References}

Ait-Sahalia, Y., Cacho-Diaz, J., Leaven, R. J. A. (2015). Modeling Financial Contagion Using Mutually Exciting

Jump Processes. Journal of Financial Economics, 117(3), 586-606, https://doi.org/10.1016/j. jfineco.2015.03.002

Andersen, T. G., Bollerslev, T., Diebold, F. X., Vega, C. (2007). Real-Time Price Discovery in Global Stock, Bond and Foreign Exchange Markets. Journal of International Economics, 73(2), 251-277, https://doi.org/10.1016/j.jinteco.2007.02.004

Bandi, F. M., Reno, R. (2016). Price and Volatility Co-Jumps. Journal of Financial Economics, 119(1), 107-146, https://doi.org/10.1016/j.jfineco.2015.05.007

Barndorff-Nielsen, O. E., Shephard, N. (2004). Power and Bipower Variation with Stochastic Volatility and Jumps. Journal of Financial Econometrics, 2(1), 1-37, https://doi.org/10.1093/ jjfinec/nbh001

Behfahr, S. K. (2016). Long Memory Behavior of Returns After Intraday Financial Jumps. Physica A, 461, 716-725, https://doi.org/10.1016/j.physa.2016.06.026

Brazys, J., Martens, M. (2015). How Important is Economic News for Bond Markets? Erasmus University of Rotterdam. Working Paper, https://doi.org/10.2139/ssrn.2311393

Brazys, J., Duyvesteyn, J., Martens, M. (2015). Macroeconomic News and Price Discovery in International Bond Markets. European Financial Management Association 2015 Annual Meetings, Amsterdam. 
Corsi, F., Pirino, D., Reno, R. (2010). Threshold Bipower Variation and the Impact of Jumps on Volatility Forecasting. Journal of Econometrics, 159(2), 276-288, https://doi. org/10.1016/j.jeconom.2010.07.008

Dumitru, A. M., Urga, G. (2012). Identifying Jumps in Financial Assets: A Comparison Between Nonparametric Jump Tests. Journal of Business \& Economic Statistics, 30(2), 242-255, https://doi.org/10.1080/07350015.2012.663250

Fičura, M. (2015). Modelling Jump Clustering in the Four Major Foreign Exchange Rates Using High-Frequency Returns and Cross-exciting Jump Processes, Procedia Economics and Finance, 25, 208-219, https://doi.org/10.1016/S2212-5671(15)00731-5

Fičura, M., Witzany, J. (2016). Estimating Stochastic Volatility and Jumps Using High-Frequency Data and Bayesian Methods. Czech Journal of Economics and Finance, 66(4), 279-301.

Fulop, A., Li, J., Yu, J. (2014). Self-Exciting Jumps, Learning, and Asset Pricing Implications. Review of Financial Studies, 28(3), 876-912, https://doi.org/10.1093/rfs/hhu078

Hanousek, J., Kočenda, E., Novotný, J. (2011). The Identification of Price Jumps. CERGE-El. Working Paper Series No. 434, ISSN 1211-3298, https://doi.org/10.2139/ssrn.1784405

Jiang, J. G., Oomen, R. C. A. (2008). Testing for Jumps when Asset Prices are Observed with Noise - a "Swap-Variance“ Approach. Journal of Econometrics, 144(2), 352-370, https://doi.org/10.1016/j.jeconom.2008.04.009

Lee, S. S., Mykland, P. A. (2008). Jumps in Financial Markets: A New Nonparametric Test and Jump Dynamics. Review of Financial Studies, 21(6), 2535-2563, https://doi.org/10.1093/rfs/ hhm056

Novotny, J., Petrov, D., Urga, G. (2015). Trading Price Jump Clusters in Foreign Exchange Markets. Journal of Financial Markets, 24, 66-92, https://doi.org/10.1016/j.

finmar.2015.03.002 